\title{
ESTUdO ANATÔMICO dOS PLEXOS VENOSOS VERTEBRAIS NO CÃO*
}

\author{
STUDY ANATOMY OF THE VERTEBRAL VENOUS SYSTEM IN THE DOG
}

\author{
Domingos Jose Sturion**
}

\section{RESUMO}

O autor descreve a anatomia dos plexo venoso vertebral, cujo perfeito conhecimento é fundamental para uma correta interpretação da venografia epidural intra-óssea e flebografia epidural.

Palavras-chave: anatomia, plexo venoso vertebral, cão, coluna vertebral.

\section{SUMMARY}

The author describes the anatomy of the vertebral venous system. It is fundamental to know the correct intraosseus and epidural phlebography to make right interpretation.

Key words: anatomy, vertebral venous system, dog, vertebral column.

\section{INTRODUÇÃO}

O sistema venoso vertebral constitui uma rota alternativa de retorno do sangue para o coração, pelas anastomoses com o sistema das veias anteriores e ázigos, estando o sangue sujeito a fluir em sentido cranial ou caudal dependendo da pressão relativa (BATSON, 1957; MILLER et al, 1964).

Vesalius, apud ISHERWOOD (1962), foi o primeiro a observar os ramos das veias vertebrais entrando no canal vertebral, entretanto o conceito de um sistema das veias vertebrais é creditado a Breschet, apud BATSON (1957). Este autor foi o primeiro a apreciar plenamente a complexicidade destas veias com o crânio e a coluna vertebral, apresentando um estudo adequado e complexo das veias vertebrais.
Muitos dos conhecimentos atuais devem-se a BATSON (1940, 1957) que chamou a atenção, através de seus estudos com técnicas de injeção/corrosão em cadáveres humanos, para a importância do sistema venoso vertebral na disseminação de infecções e metástases, classificando-os e dividindo-os em dois grupos principais de acordo com a localização: (1)veias no interior da cavidade tóraco-abdominal: veias cava, pulmonar, portal e lombo-ázigos. (2) veias fora da referida cavidade; todas emitindo colaterais para o interior da coluna vertebral, formando o sistema de veias vertebrais.

As primeiras tentativas de visualização do plexo venoso vertebral in vivo, todavia, foram realizadas por FARINAS (1947) e ANDERSON, em 1951, tendo este último após injeção de contraste em uma veia femoral sob compressão da veia cava caudal, "duplicado em detaIhes" as observações de BATSON (1940, 1957), contribuindo para o conhecimento da anatomia desse sistema.

O sistema venoso vertebral está constituído de um conjunto de veias avalvulares, que drenam a medula vertebral, as raízes nervosas, meninges, corpos e arcos vertebrais posteriores e músculos paravertebrais. Nesses vasos, que têm paredes finas e delgadas, desprovidos de fibras musculares, e facilmente distensíveis, o sangue flui livremente por gradiente de pressão (ECKENHOFF, 1970; SANTOS, 1985). O conhecimento da anatomia desse sistema, formado por uma complexa rede que percorre toda a extensão da coluna vertebral - da cauda até a base do occiptal - é fundamental para uma correta interpretação da venografia epidural intra-óssea e flebografia epidural. De acordo com a sua localização dentro e fora do canal vertebral, esse sistema pode ser dividido em dois plexos - plexo venoso vertebral interno e externo, que estão interligados em múltiplos níveis.

\section{DESENVOLVIMENTO}

Plexo venoso vertebral externo

O plexo venoso externo ou paravertebral é sub-

\footnotetext{
* Trabalho realizado com auxilio do CNPq.

** Médico Veterinário, Doutor, Professor do Departamento de Clínicas Veterinárias da Universidade Estadual de Londrina, Caixa Postal 6001. 86051-970 LONDRINA-PR.
} 
dividido em quatro partes, correlacionadas com o segmento espinhal correspondente e está representado pelas veias ilíacas internas, externas e sacral mediana na pélvis (Figura 1), veias ázigos e hemiázigos no tórax e veias vertebrais no pescoço (Figura 2).

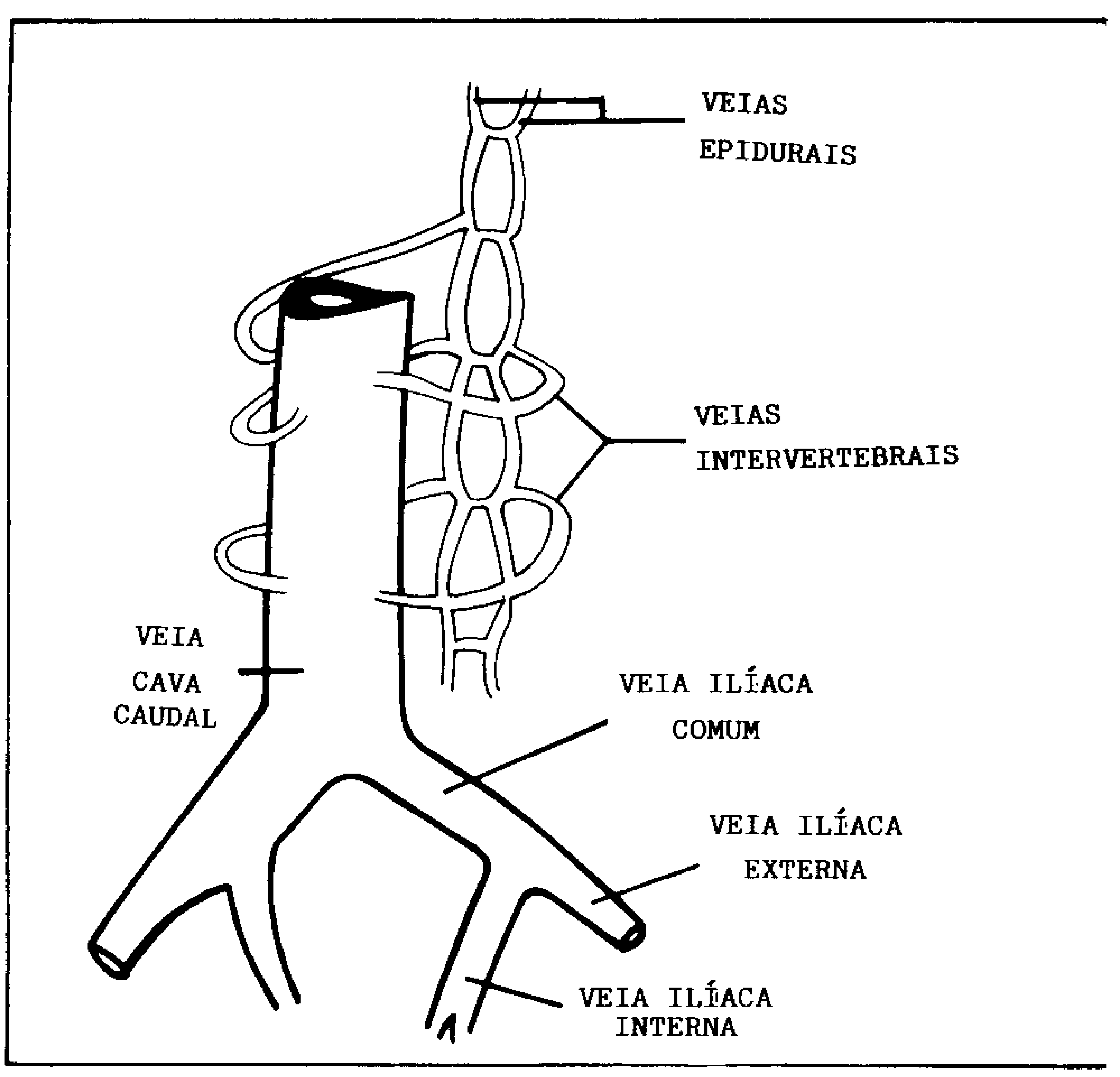

FIGURA 1 - Representaçāo diagramática das veias epidurais, veias intervertebrais, veia cava caudal, veias ilíacas.

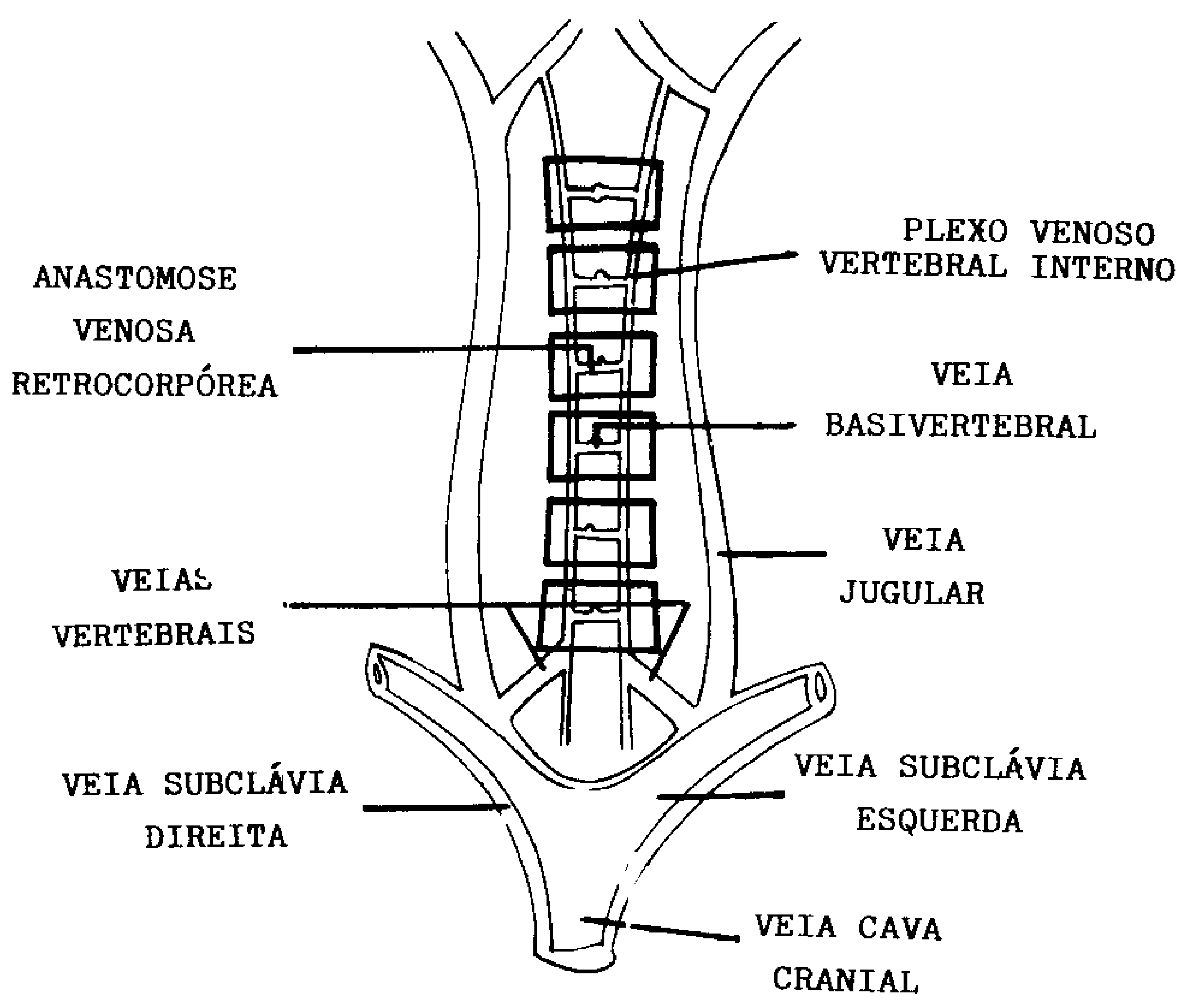

FIGURA 2 - Representaçāo diagramática do plexo venoso vertebral (interno e externo) na região cervical
Veia illaca interna

As veias iliacas internas ou veias hipogástricas direita e esquerda, originam-se à altura da articulação sacro-ilíaca pela confluência das veias sacrais laterais, glúteas e pudendas internas, drenam os órgãos pélvicos e comunicam-se através das veias sacrais laterais com o plexo venoso sacral. Geralmente mediais as artérias correspondentes, unem-se as veias ilíacas externas homolaterais para formar as veias ilíacas comuns. A veia obturatriz pode também abrir-se em uma delas. São troncos curtos e mais pequenos que as veias iliacas externas. Uma rama anastomótica pōe em conexão a veia pudenda interna e a veia obturatriz a nível da escotatura ciática menor. As veias pudendas internas recebem afluentes do ânus, e da parte posterior do reto, do plexo venoso do prepúcio e do pênis do macho, e do plexo venoso da glândula mamária, vulva e vestíbulo na fêmea (MILLER et al, 1964).

Variações anatômicas podem ocorrer nas veias ilíacas internas como: presença de dois troncos em um só lado, com um deles drenando na veia ilíaca comum heterolateral; ausência de comunicaçāo com a veia ilíaca comum correspondente, dando-se a drenagem na veia ilíaca comum do outro lado; junção com a veia ilíaca externa em local fora do habitual (acima e abaixo), duplicação uni ou bilateral, curso anormal (SANTOS, 1985).

\section{Veia ilíaca externa}

Surge da porção lateral da veia cava caudal, normalmente entre a sexta e sétima vértebra lombares.

A veia ilíaca externa corre obliquamente no sentido ventrocaudal, primeiro ao longo do tendão do músculo iliopsoas até a borda cranial do osso pélvico, onde deixa a cavidade abdominal ao passar através do ângulo femural. O principal ramo da veia ilíaca externa é a veia femoral profunda que drena o membro posterior.

\section{Veia sacral mediana}

Desprovida de tributárias significantes, é única e recebe a veia caudal média desde a cauda. Envia ramos entre o músculo sacrocaudal direito e esquerdo e termina, de acordo com WORTHMAM (1956), em ambas veias ilíacas comuns direita e esquerda.

Esta é uma pequena veia de cerca de $1 \mathrm{~mm}$ de diâmetro usualmente desprovida de tributárias significantes. Em algumas espécies estas são largas e coletam tributárias curtas e simples das duas primeiras veias intervertebrais direita e esquerda e os últimos pares das 
veias intervertebrais lombares. Após estas são únicas com tronco comum (MILLER et al, 1964).

KOBLIK \& SUTER (1981), utilizando a cateterização transfemoral percutânea até a veia sacral mediana, observaram o plexo venoso vertebral interno, e a variação e localização desta veia assim como a presença de válvulas em sua terminação, as quais foram confirmadas pela dissecção (Figura 3).

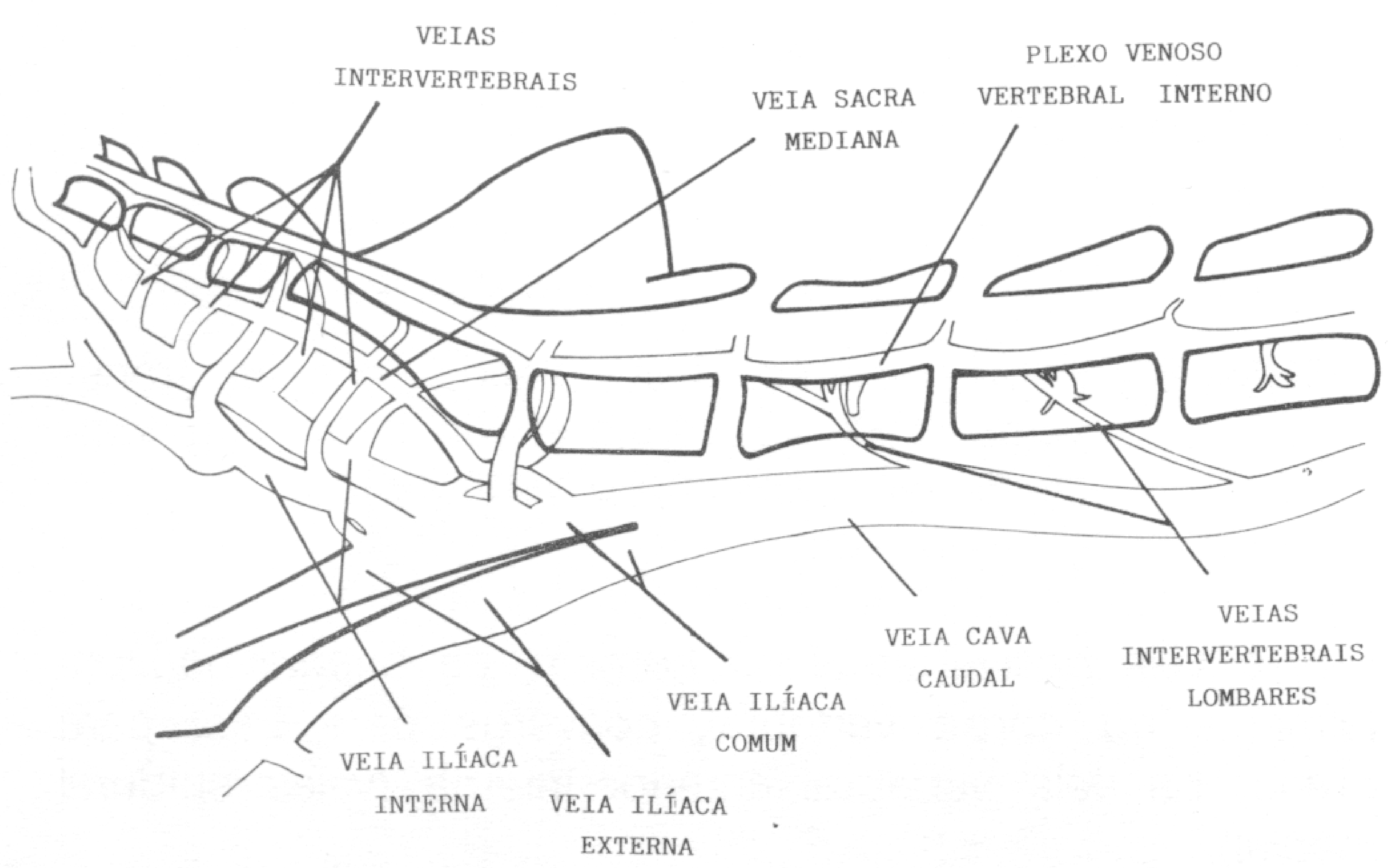

FIGURA 3 - Representação diagramática do plexo venoso vertebral (interno e externo) na região lombo-sacral.

Veia ázigos

Apresenta cerca de $8 \mathrm{~mm}$ de diâmetro em sua junção com a veia cava cranial, onde a última termina no átrio direito, oposta ao primeiro espaço intercostal direito. Esta se inicia do plano medial ventral do corpo da primeira vértebra lombar pelas anastomoses com os troncos simples formados pela emergência das primeiras veias intervertebrais lombares direita e esquerda (Figura 4).

Plexo venoso vertebral interno

O plexo venoso vertebral interno ou veias epidurais situa-se dentro do canal vertebral entre a duramáter e o assoalho do canal epidural, recebendo ramos da medula vertebral e do corpo vertebral e vão desde o forame magno até a quarta e sexta vértebras caudais.

O plexo venoso vertebral interno no cão é constituído basicamente por duas veias longitudinais, cada uma delas em estreita correlação com o pedículo vertebral.

O sistema venoso vertebral do cão é semelhante ao do homem, entretanto, comparativamente possui formação e ramificações mais simples. Enquanto que no homem este sistema está constituído basicamente por



FIGURA 4 - Radiografia na incidência lateral, observando-se o perfeito preenchimento do plexo venoso vertebral interno(a), veias intervertebrais(b) e a veia ázigos(c) a nível torácico.

dois pares de veias longitudinais anteriores (veias epidurais mediais e veias epidurais laterais), cada um deles em estreita correlação com o pedículo vertebral (SANTOS, 1985), no cão existe apenas um par venoso (plexo venoso vertebral interno). Estes troncos são simétricos, de paredes finas com achatamento e sem válvulas, se desviam lateralmente ao nível de cada espaço de disco intervertebral e medialmente ao nível do pedículo elas se juntam assumindo, assim, uma configuração hexagonal (Figura 5 e 6). Seu diâmetro é reduzido na junção da última vértebra cervical e primeira torácica, com ramos constante até o plano da quarta e quinta vértebra lombar. Caudais a este plano diminuem de tamanho, podendo fundir-se no interior da quarta para sexta vértebras coccígeas ou terminas em finas venulas na musculatura caudal no interior do canal (MILLER et al, 1964; STURION, 1990) (Figura 7).

\section{Veias do corpo vertebral}

As veias basivertebrais foram descritas por Breschet, apud BATSON (1957), como provenientes da cavidade óssea da vértebra, para unir-se ao circulus vasculosus, entre a coluna vertebral e a dura-máter (Figura 8).

Com a finalidade de melhor avaliar os vasos intra-ósseos do corpo vertebral, assim como o plexo venoso subcondral adjacente ao disco intervertebral, CROCK et al (1973) realizaram um minucioso estudo em cadáver. Neste trabalho após injeção de substância radiopaca, durante necropsia em artérias e veias de 100 colunas vertebrais, as radiografias obtidas mostraram 


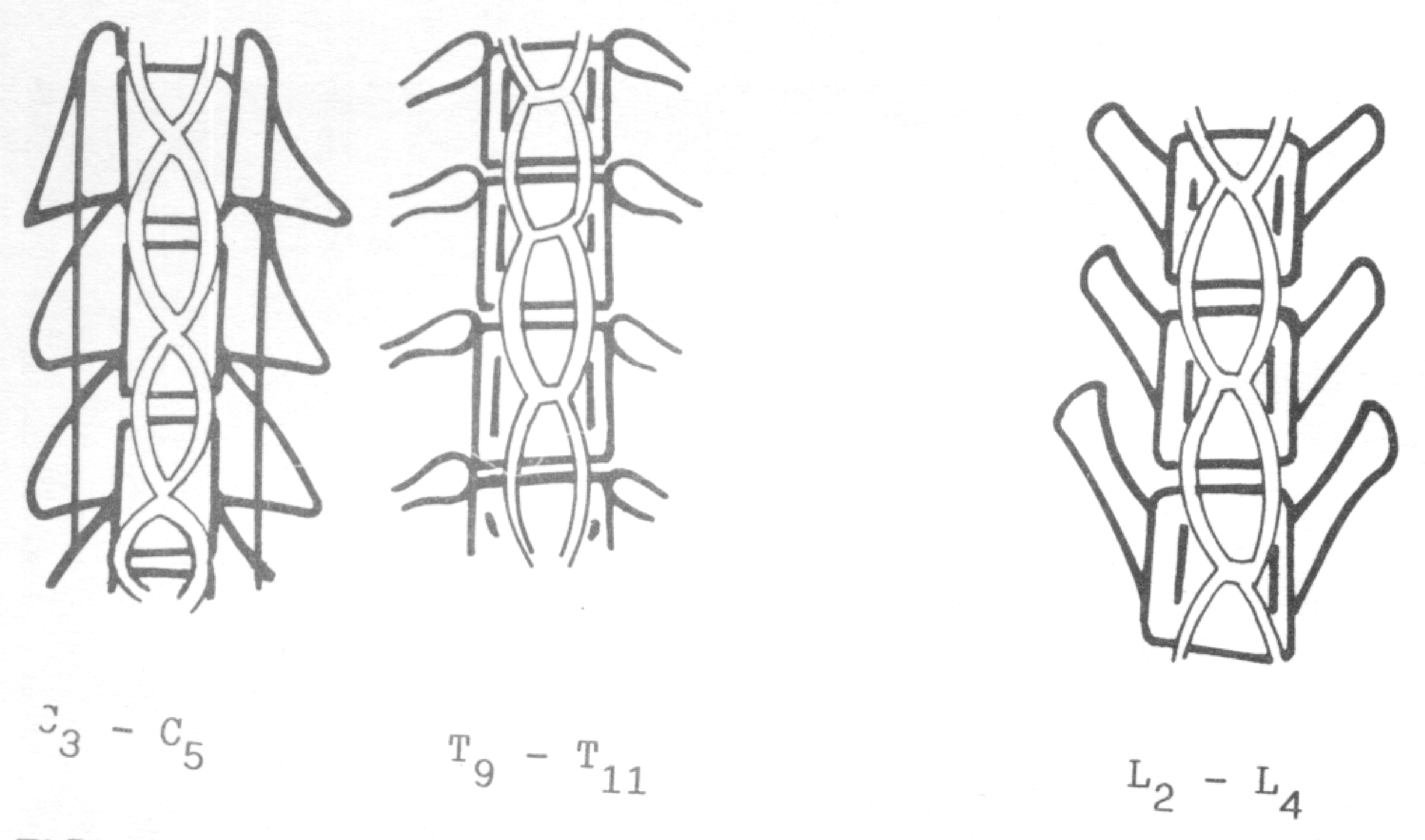

FIGURA 5 - Venograma normal dos plexos venosos vertebrais internos, na incidência ventro-dorsal.

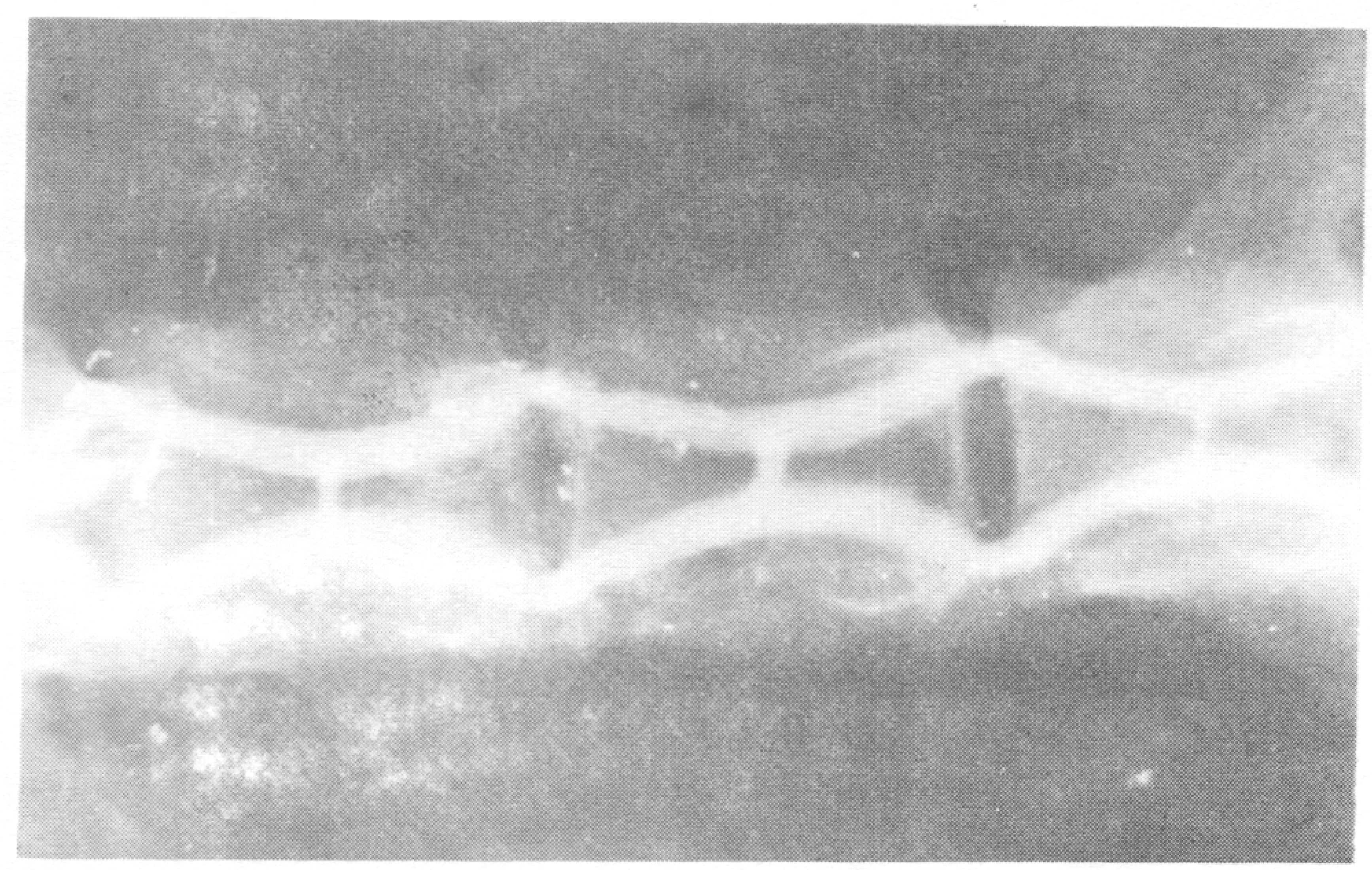

FIGURA 6 - Perfeito preenchimento dos plexos venosos vertebrais internos, com presença de anastomoses entre ambos.

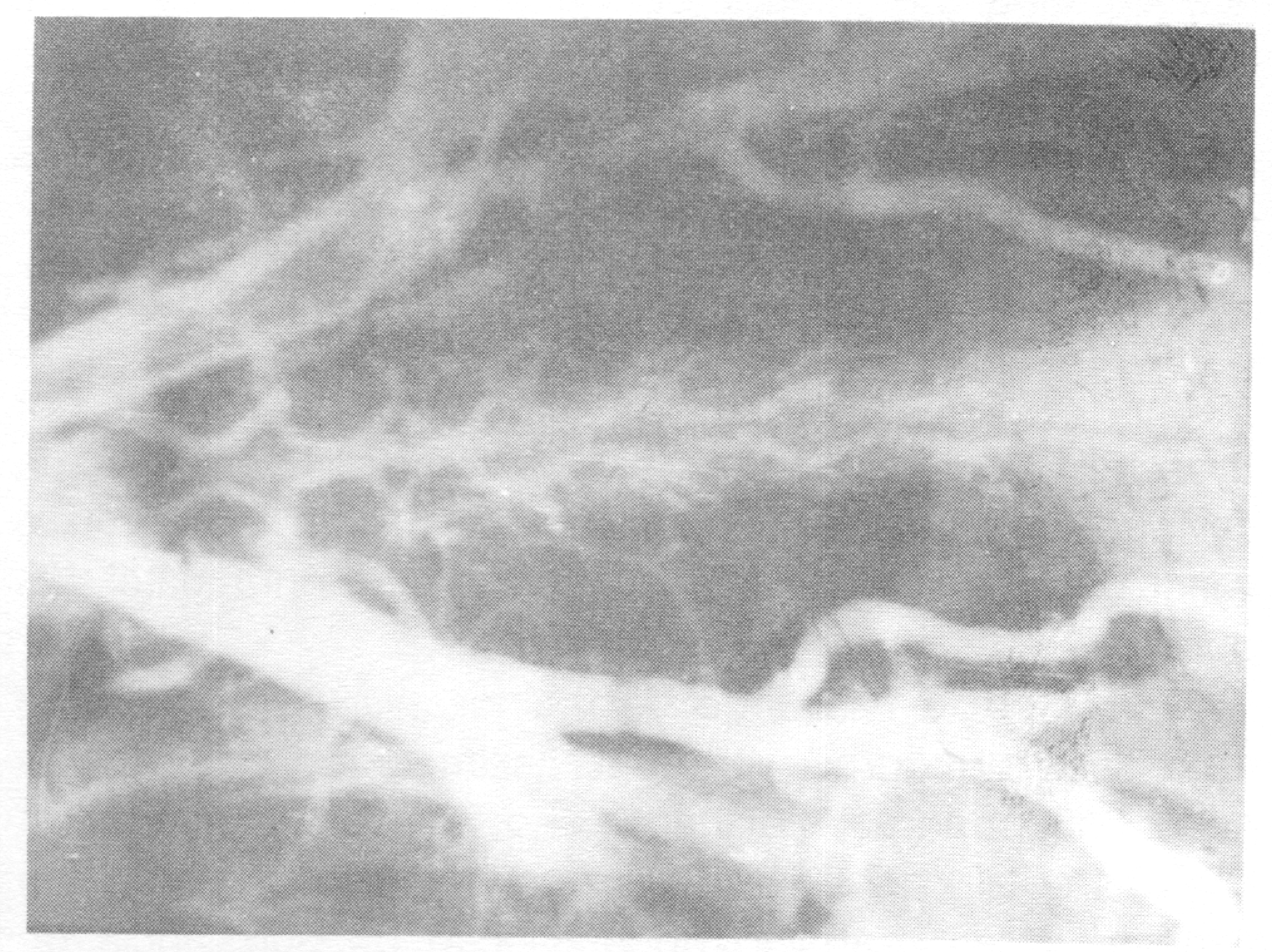

FIGURA 7 - Plexos venosos vertebrais na região caudal.

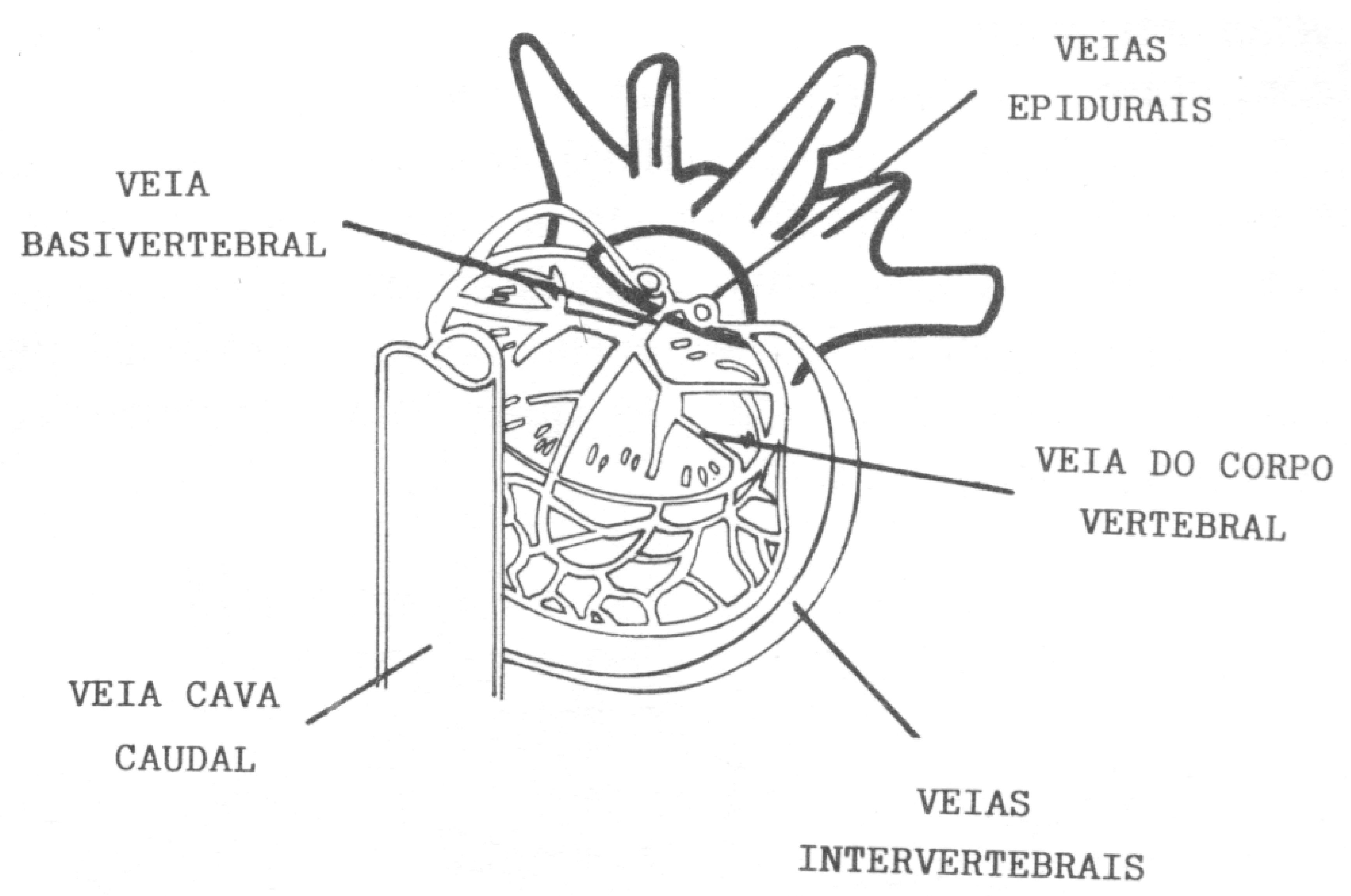

FIGURA 8 - Representação diagramática, secção transversa. Veia basivertebral (de onde originamse as veias do corpo vertebral).

que a veia basivertebral no homem, se orienta horizontalmente no corpo vertebral, converge para trás, para drenar na veia vertebral anterior interna (veias epidural medial).

Estas veias deixam 0 aspecto posterior por dois grandes forames contínuos. No segmento cranial da região torácica do cão elas não são evidentes, porém, mais caudalmente, iniciam anastomoses com as veias intercostais ( $\mathbf{v}$. intercostais dorsales). Na região lombar são extensas, usualmente pares e conectadas com as veias lombares (V. lombales) sobre o meio do plexo venoso vertebral, não se apresentando nas vértebras sacrais e coccígena (Vert. sacrales e coccigea), (MILLER et al, 1964).

O padrão das veias intra-ósseas, propriamente, é descrito como semelhante ao das raízes fibrosas das plantas comuns, a maioria delas de tamanho similar a pequenos cachos de radículas, tão finas quanto um fio de cabelo (Figura 9).

Veias intervertebrais $\mathrm{Nv}$. intervertebrales)

O plexo venoso vertebral interno é ligado, em cada espaço discal, com o plexo venoso vertebral externo, através das veias intervertebrais, sendo esta a principal conexão entre ambos. Geralmente, duas destas veias passam, através de cada forame intervertebral, paralelamente à raíz venosa emergente, uma acima e outra abaixo de cada pedículo e vão drenar diretamente no interior das veias ázigos ( $\mathbf{V}$. azygos), hemiázygos $(\mathrm{V}$. hemiazygos), espinhais, ou veia cava caudal (v. cava caudalis), no cão (PARKER, 1973), e ainda, dentro da veia lombar ascendente ( $V$. lumbales ascendens) no homem (SALEN, 1971; SANTOS, 1985). (Figura 10).

As veias intervertebrais recebem ramos afluentes 


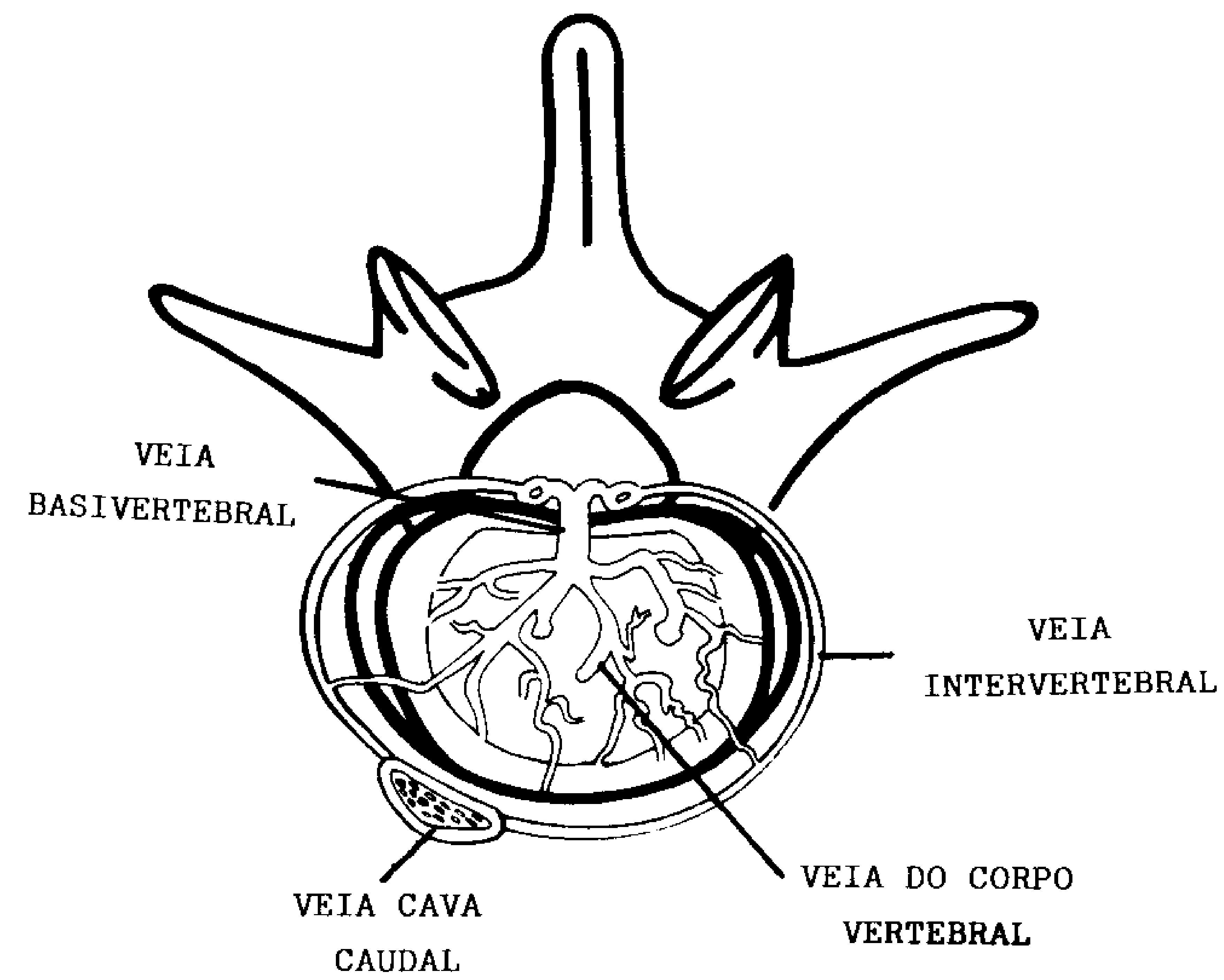

FIGURA 9 - Representação diafragmática das veias do corpo vertebral.

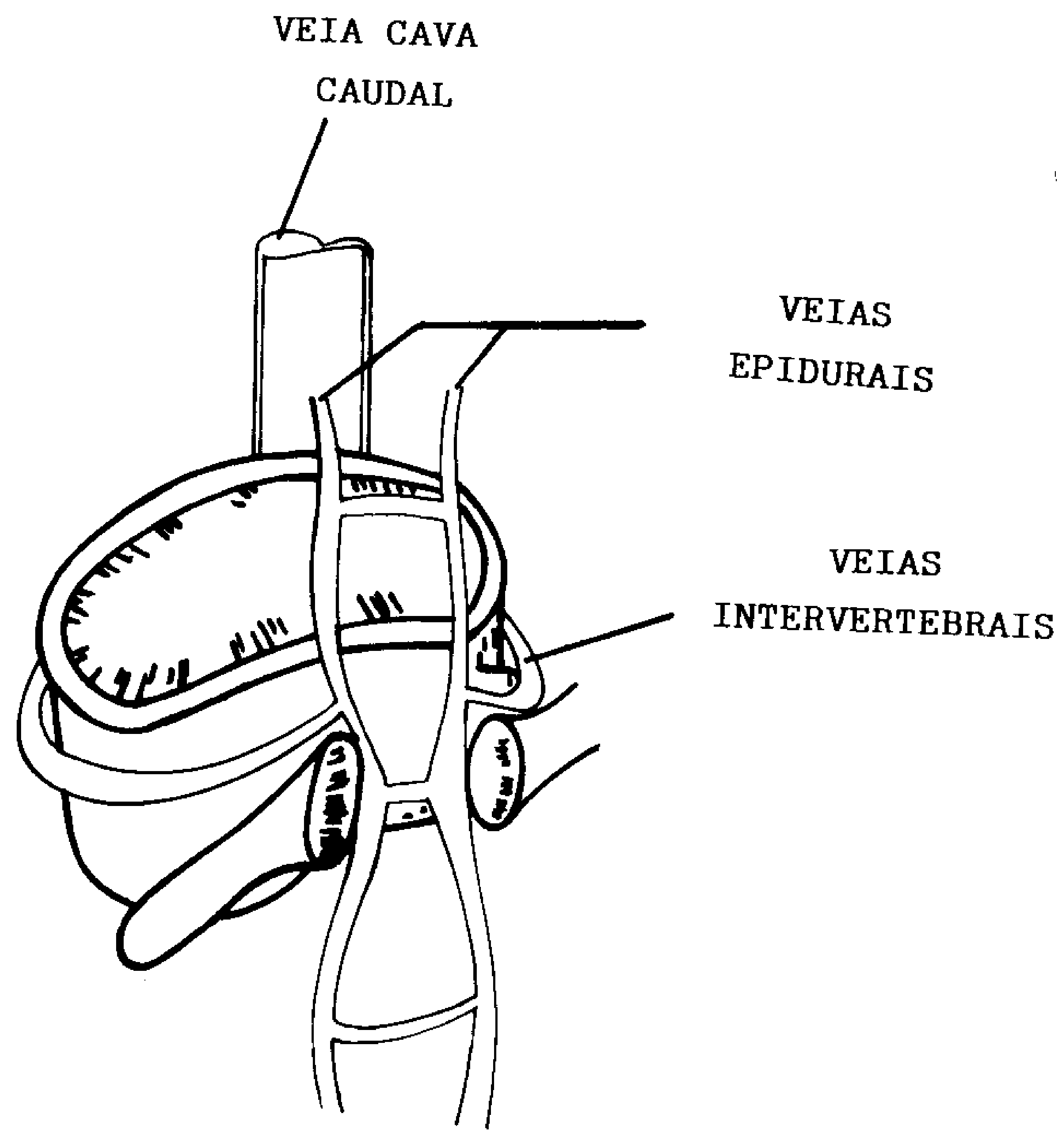

FIGURA 10 - Representação diagramática do plexo vonoso vertebral (visão VD.).

da medula espinhal (veias radiculomedulares) (GARGANO, 1980), podem ser únicas ou múltiplas, mas guardam uma simetria lateral constante e têm sido denominadas de maneira adversa pelos diferentes autores: veias radiculares (MACNAB et al, 1976), veias supra e infrapediculares (SHAPIRO et al, 1980), veias do forme lateral (CHERMET \& BIGOT, 1980), veias emissárias do forame intervertebral (ROLAND et al, 1978), veias inter- vertebrais (WILMINK et al, 1978), veias do buraco de conjugação (ROLAND et al, 1976), no homem. No cão as veias intervertebrais recebem o nome e número do espaço intervertebral através dos que passam, exceto para as duas primeiras veias intervertebrais sacrais que passam através dos forames sacro ventral de cada lado. Assim na região lombo-sacra as veias intervertebrais $\mathrm{VI}$ e VII, drenam para a veia ilíaca interna comum e a veia cava caudal. Já a veia intervertebral sacral I, drena para a veia glútea cranial, a veia intervertebral sacral, para a veia pudenda interna, a veia intervertebral caudal $I$ até a IV, para a veia sacra mediana e veia ilíaca interna (MILLER et al, 1964).

MCCORMICK (1978), distingue as veias que passam acima e abaixo do pedículo vertebral, denominando de veia discal aquela que se relaciona com a borda inferior do disco vertebral e a borda superior adjacente do corpo vertebral abaixo, e de veia pedicular a que passa abaixo do pedículo. Segundo ele, enquanto a segunda é de menor importância, a veia discal é fundamental para o diagnóstico da protrusão do disco intervertebral.

O fluxo nas veias intervertebrais é bidirecionado e está sujeito a fatores de regulação. Quando a pressão intra-abdominal está aumentada por tosse, esforço, manobra de Valsalva, por uso de compressão abdominal, no caso de anormalidade com falta de desenvolvimento da veia cava caudal, o fluxo nas veias intervertebrais vai para as veias vertebrais e ázigo. Sem o aumento da pressão o fluxo está na direção da veia cava caudal (HARRIS, 1941; MORGORE, 1945; NORDENSTROM 1955; GARGANO, 1980; Le PAGE, 1974; RENARD et al, 1976).

\section{CONCLUSÃO}

O estudo da anatomia dos plexos venosos vertebrais no cão, permitem concluir o seguinte:

- devido a complexa rede que percorre estes plexos em toda a extensão da coluna vertebral, desde a cauda até a base do occiptal é fundamental seu conhecimento para uma correta interpretação das técnicas de venografia epidural intra-óssea e flebografia epidural no cão. Apesar de constituir uma complexa rede estes vasos sāo bem visualizados no exame radiográfico.

\section{REFERÊNCIAS BIBLIOGRÁFICAS}

ANDERSON, R.R. Diotrast studies of the vertebral and cranial venous system to show their probable role in cerebral metastases. I Neurosurg, v. 8, p. 411-422, 1951. 
BATSON, O.V. Function of the vertebral veins and their role in the spread of metastases. Am Surg, Philadelphia, v. 112, p. 138-149, 1940.

BATSON, C.V. The vertebral veins system. Am J Roentgenol. Baltimore, v. 78, n. 2, p. 195-212, 1957.

CHERMET, J., BIGOT, J.M. Venography of the inferior vena cava and this branches. Berlin: Springer-Verlag, 1980. p. 197.

CROCK, H.V., YOSHIZAWA, H., KAME, S.W. Observations on the venous drainage of the vertebral body. Bone Joint Surg, v. 55-B, n. 3, p. 528-533, 1973.

ECKRNHOFF, J.E. The physiologic significance of the vertebral venous plexus. Surg Gynecol Obstet, v. 131, n. 1, p. 72-78, 1970.

GARGANO, E.P. Extradural venography. In: POST, J.M. D., Radiographic Evaluation of the Spine. Current advances with emphasis on computed tomography. New York: Masson Publishing, 1980, p. 579-592.

HARRIS, H.A. A note on the clinical anatomy of the veins, with special reference to the spinal veins. Brain, London, v. 64, p. 291-300, 1941.

ISHERWOOD, I. Spinal intra-osseous venography. Clin Radiol Edinburg, v. 13, p. 73-82, 1962.

KOBLIK, P., SUTER, P.F. Lumbo-sacral vertebral sinus venography via transjugular catheterization in the dog. Vet Radiol, Hagerstown, v. 22, n. 2, p. 69-77, 1981.

LePAGE, J.R. Transfemoral ascending lumbar catheterization of the epidural veins. Exposition and technique. Radiology, v. 111, p. 337-339, 1974.

MACNAB, L., St. LOUIS, E.L., GRABIAS, S.L., et al. Seletive ascending lumbosacral venography in the assessment of lumbar-disc herniation. Bone Joint Surg. v. 58-A, n. 8, p. 1093, 1976.

MCCORMICK, C.C. Radiology in lowback pain and sciatica. An analysis of the relative efficacy of spinal venography, discography and epidurography in patients with a negative or equivocal myelogram. Clin Radiol, v. 29, p. 393-406, 1978.

MILLER, M.E., CRISTENSEN, G.C., EVANS, H.E. Anatomy of the dog. Philadelphia: Saunders, 1964. cap. 5: The venous system: p. 389-429.

MORGORE, $M$. Clinical anatomy of the vertebral veins. Surgery, St. Louis, v. 17, p. 66-615, 1945
NORDENSTROM, B. A method of angiography of the azigos vein and the anterior interior plexis of the spine. Acta Radiol. Finland, v. 44, p. 201-208, 1955.

PARKER, J.A. Clinical significance of traumatic occlusion of segmental spinal arteries. J Am Vet Med Assoc, Chicago, v. 162, n. 12, p. 1041-1042, 1973.

RENARD, M., MASSON, J.P., LARDE, D. Les veines epidurales lombaires (Etude anatomique à propus de 31 cas). Bull Ass Anat, v. 60, n. 171, p. 787-800, 1976.

ROLAND, J., LARDE, D., SCHWARTZ, J.F., et al. Intéret de la phlebographie dans le diagnostic des discopathies lombaires. J Radiol Eletrol, v. 57, n. 2, p. $175-182,1976$

ROLAND, J., TREIL, J., LARDE, D., et al. Lumbar phlebography in the diagnosis of disc herniations. Neurosurg, v. 49, p. $544-550,1978$.

SALEN, E.F. A phlebography study of constructive processes in the superior vena cava and of accompanhying changes in the collateral circulation. Acta Radiol. Stockolm, v. 36, p. 81-87, 1971.

SANTOS, M.B. Contribuiçăo da flebografia epidural ao diagnóstico da hérnia discal lombar; estudo comparativo com a radiculografia Rio de Janeiro, 1985. 155 p. Dissertação (Mestrado em Medicina). Faculdade de Medicina da UFRJ, 1985.

SHAPIRO, K., TADAVARTHY, S.M., GORDONS, S. Role of transfemoral lumbar epidural venography in the diagnosis of herniated lumbar discs. Min Med, v. 63 , n. 9, p. 639-643, 1980.

STURION, D.J. Análise comparativa das técnicas de venografia epidural intra-6ssea $\theta$ flebografia transfemoral em cães, realizadas com bloqueio do fluxo sangüíneo da veia cava caudal. Botucatu, 1990. 47 p. Tese (Doutorado em Medicina Veterinária, área clínica). Universidade Est. Paulista, Júlio de Mesquita, Campus Botucatu, 1990

WILMINK, J.T., PENNING, L., BENKS, J.W.F. Techniques in transfemoral lumbar epidural phlebography. Neuroradiology, v. 15, p. 273-286, 1978.

WORTHMAN, R.P. The longitudinal vertebral venous sinuses of the dog: Anatomy: II Functional aspects. Am J Vet Rev, v. 17, p. 341-363, 1956. 